\title{
Phenotypic Screening of Aminoglycoside Resistance and their Transferability in Clinical Isolates of Klebsiella pneumoniae from India
} Nargis Alom Choudhury ${ }^{1}$, Sunanda Deb ${ }^{1}$, Anand Prakash Maurya ${ }^{1}$, Debadatta Dhar Chanda ${ }^{2}$, Atanu Chakravarty ${ }^{2}$ and Amitabha Bhattacharjee ${ }^{1 *}$ ${ }^{1}$ Department of Microbiology, Assam University, Silchar, Assam, India

${ }^{2}$ Department of Microbiology, Silchar Medical College and Hospital, Silchar, Assam, India

\begin{abstract}
Introduction: Klebsiella pneumonia is an emerging pathogen associated with multidrug resistance both in hospital and community settings. Aminoglycosides, considered to be second line drug for the treatment of such pathogens, become inactive due to acquisition of various resistance determinants by this organism.

Objective: The objective of the study was to screen the aminoglycoside resistant Klebsiella pneumonia from a tertiary referral hospital of northeast India and their transmission dynamics.

Method: A total of 177 consecutive, non-duplicate, clinical isolates of Klebsiella pneumonia were collected from patients from a period of September 2013 to February 2014. Screening for aminoglycoside resistance was performed. Transferability of aminoglycoside resistance was done by transformation assay. Genetic stability was checked by consecutive serial passage of 70 days. Incompatibility types were determined by PCR based replicon typing.

Result: Among 177 clinical isolates, 94 were screened to be resistant towards aminoglycoside group of antibiotics. The aminoglycoside resistance determinant was found to be transferable when transformants were selected in gentamicin $(100 \mu \mathrm{g} / \mathrm{ml})$ screen agar. Coresistance was also shown by these isolates. Gentamicin resistance was lost after 47 consecutive serial passages. $F$ inc type $(n=17)$ was more predominant, followed by K/B $(n=11), Y(n$ $=13), I(n=9)$ and $P(n=8)$ when plasmids were typed by PCR based replicon typing.
\end{abstract}

Conclusion: This study highlighted the transmission dynamics of aminoglycoside resistance determined which pose threat to the treatment option in hospital settings.

Keywords: Multidrug resistance; Aminoglycosides; Transferability; Klebsiella pneumoniae

\section{Introduction}

Klebsiella is known to be the second most important multidrug resistant organism within enterobacteriaceae family after Escherichia coli. Klebsiella pneumoniae often reported to produce carbapenemase (KPC) leaving the carbapenem antibiotics inactive on them. The organism too often exhibit resistance towards quinolone and aminoglycoside group of antibiotics [1] that continue to play an important role in antimicrobial therapy against both gram negative and gram-positive pathogens, usually in combination with $\beta$-lactam agents [2]. Resistance to aminoglycosides can be widespread and has primarily been the result of aminoglycoside inactivation through the chemical processes of acetylation, phosphorylation, and/or adenylation, with varying effects depending upon the particular agent $[3,4]$. Recently another mechanism i.e, acquired $16 \mathrm{~S}$ methyl transferase is also known to play a significant role in aminoglycoside resistance. These mechanisms, when combined with additional coexisting multidrug resistant trait, pose severe threat to therapeutic regime. Till now there is a paucity of data regarding aminoglycoside resistance among gram negative pathogen from this geographical part of the world. Thus, this study was undertaken to screen aminoglycoside resistance and their transferability within member of enterobacteriaceae family.

\section{Methods}

\section{Sample size}

A total of 177 consecutive, non- duplicate, clinical isolates of Klebsiella pneumoniae were collected from indoor and outdoor patients of Silchar Medical College and Hospital, India for duration of six month from September 2013 to February 2014. Isolates were identified based on conventional microscopical observation, cultural characteristics and biochemical testing methods.

\section{Phenotypic screening of multidrug resistant Klebsiella}

Detection of susceptibility in isolates was performed by Kirby bauer disc diffusion method using following antibiotics ciprofloxacin $(10 \mu \mathrm{g})$, gentamicin $(10 \mu \mathrm{g})$, amikacin $(30 \mu \mathrm{g})$, meropenem $(10 \mu \mathrm{g})$, cotrimoxazole $(25 \mu \mathrm{g})$, netilimicin $(10 \mu \mathrm{g})$ and kanamicin $(10 \mu \mathrm{g})$ (Hi-Media, Mumbai, India). E. coli ATCC 25922 was used as quality control strain. Antibiotic susceptibility testing was performed for both wild type and transformants for aminoglycoside group of antibiotic i.e, gentamicin $(10 \mu \mathrm{g})$, kanamycin $(30 \mu \mathrm{g})$, netilimicin $(30 \mu \mathrm{g})$, and amikacin $(30 \mu \mathrm{g})$. Results were interpreted according to CLSI guidelines [5].

\section{Transferability}

Isolated plasmids were tested for their ability of horizontal transfer by transformation assay. The recipient strain used was E. coli JM107. Transformation was carried out by heat shock method [6].

*Corresponding author: Amitabha Bhattacharjee, Department of Microbiology, Assam University, Silchar, Assam, India, Tel: 918471939088; E-mail: ab0404@gmail.com

Received October 20, 2015; Accepted February 06, 2016; Published February 11 2016

Citation: Choudhury NA, Deb S, Maurya AP, Chanda DD, Chakravarty A et al. (2016) Phenotypic Screening of Aminoglycoside Resistance and their Transferability in Clinical Isolates of Klebsiella pneumoniae from India. J Med Microb Diagn 5: 218. doi:10.4172/2161-0703.1000218

Copyright: $\odot 2016$ Choudhury NA, et al. This is an open-access article distributed under the terms of the Creative Commons Attribution License, which permits unrestricted use, distribution, and reproduction in any medium, provided the original author and source are credited. 
Citation: Choudhury NA, Deb S, Maurya AP, Chanda DD, Chakravarty A, et al. (2016) Phenotypic Screening of Aminoglycoside Resistance and their Transferability in Clinical Isolates of Klebsiella pneumoniae from India. J Med Microb Diagn 5: 218. doi:10.4172/2161-0703.1000218

Page 2 of 3

Transformants were selected on LB Agar plates containing gentamicin $(0.25 \mu \mathrm{g} / \mathrm{ml})$.

\section{Analysis of stability of plasmid encoding multidrug resistant phenotype}

The experiment was performed to determine the vertical transmission dynamics of resistant genes along with their plasmid in subsequent generations. Plasmid stability of screened multi drug resistant (MDR) isolates as well as their transformants was analyzed by serial passages method for consecutive 75 days at 1:1000 dilutions without antibiotic pressure [7].

\section{Determination of plasmid incompatibility typing}

Plasmid incompatibility was determined for wild type as well as transformants by PCR based replicon typing using 18 pairs of different basic replicon primers, targetting FIA, FIB, FIC, HI1, HI2, I1-Ig, L/M, N, P, W, T, A/C, K, B/O, X, Y, F, and FIIA replicons [8].

\section{Result}

On susceptibility testing, 94 (53.6\%) organisms out of 177 , all of which were isolated from urine and pus (Table 1) were found to be resistant against one of the aminoglycosides, (gentamicin, netilimicin, amikacin, kanamycin) (Table 2) or more antibiotic. All the isolates showed multidrug resistance (MDR) phenotypes. Aminoglycoside resistance was horizontally transferrable. The plasmid could be selected on E. coli JM 107 on Luria Bertani agar containing gentamicin. Most of the transformants showed co-resistance to other representatives of aminoglycoside antibiotics (Table 3).

Transformants retained resistance till 70th passage. Gentamicin resistance was lost after 47 th passages.

Incompatibility typing could reveal that plasmid encoding aminoglycoside resistance was carried within diverse type of inc group. Among them $\mathrm{F}$ inc type $(\mathrm{n}=17)$ was more predominant,

\begin{tabular}{|c|c|}
\hline \multicolumn{2}{|c|}{ Type of Clinical specimen } \\
\hline Urine & 94 \\
\hline Pus & 83 \\
\hline
\end{tabular}

Table 1: Types of clinical specimen obtained in the study.

\begin{tabular}{|c|c|c|c|}
\hline SI.no. & Antibiotic & Number & Percentage \\
\hline 1 & Gentamicin & 20 & 18.8 \\
\hline 2 & Netilimicin & 23 & 21.62 \\
\hline 3 & Amikacin & 25 & 23.50 \\
\hline 4 & Kanamycin & 26 & 24.44 \\
\hline
\end{tabular}

Table 2: susceptibility testing against aminoglycoside group of antibiotic.

\begin{tabular}{|l|l|l|}
\hline Screen agar & No. of transformants & Co-harbouring antibiotic \\
\hline Ampicillin $(100 \mu \mathrm{g} / \mathrm{ml})$ & 70 & $\begin{array}{l}\mathrm{NET}=2, \mathrm{GEN}=3, \mathrm{KAN}=4, \\
\mathrm{TGC}=3, \mathrm{FEP}=3, \mathrm{MER}=3\end{array}$ \\
\hline
\end{tabular}

FEP: Cefipime, AMP: Ampicillin, GEN: Gentamicin, KAN: Kanamicin, MER: Meropenem, NET: Netilimicin, TGC: Tigicycline.

Table 3: Screening result of transformants.

\begin{tabular}{|c|c|}
\hline Incompatible types & Number \\
\hline F (FIA, Frep, FIC) & 17 \\
\hline K/B & 11 \\
\hline P & 8 \\
\hline Y & 13 \\
\hline I & 9 \\
\hline
\end{tabular}

Table 4: Different incompatible types.

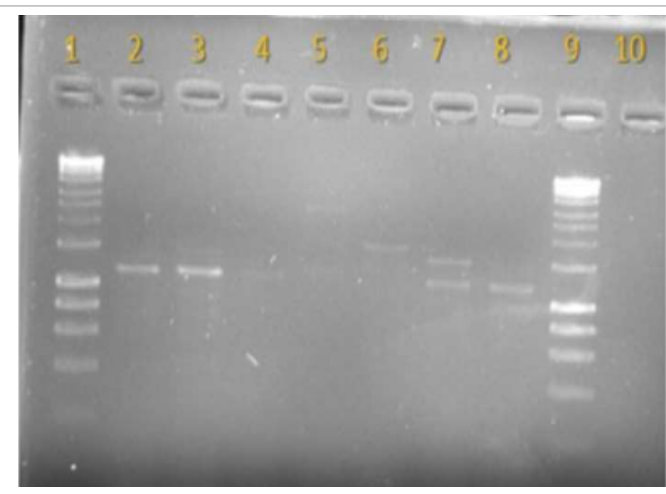

Figure 1: PCR based replicon typing of transformants, Lane 1 and 9 is hyper ladder, lane 2 and 3 showing Inc F, in lane 4 Inc $P$ and in lane 5 and 6 Inc.

followed by K/B ( $\mathrm{n}=11), \mathrm{Y}(\mathrm{n}=13), \mathrm{I}(\mathrm{n}=9)$ and P $(\mathrm{n}=8)$ (Table 4 and Figure 1).

\section{Discussion}

In recent years $K$. pneumoniae is 84 a major organism which is said to be an etiological agent in hospital and community acquired infection [9]. However, increasing resistance to aminoglycosides is becoming a serious clinical problem. Resistance to aminoglycosides is frequently due to the acquisition of modifying enzymes that vary in their substrate ranges, such as acetyltransferases, phosphorylases and adenylyltransferases. Aminoglycoside resistant klebsiella pneumonia was reported in 2009 [10].

In this study high antibiotic resistance pattern was observed within study isolates family whereas, in this study moderate resistance was observed compared to data of previous studies Jones Goosen, Scwaber. This study underscores the presence and persistence of multidrug resistance within single phenotype, with implications on clinical outcome.

Earlier it was described that plasmid transfer happens due to possible high frequencies present in host, original host carrier, which are not compatible and permits the entry of plasmids. The range of pathogens in which multi-drug resistance or virulence plasmids are stably maintained could thus expand in the future and a better understanding of the evolutionary mechanisms of plasmid host range shifts [11]. In the current study it was observed various multidrug resistance plasmids were highly stable and retained the resistance phenotype till 47 consecutive serial passages. This implies the ability of pathogens to maintain the resistance gene even there is no antibiotic exposure. Thus, therapeutic alternative becomes limited which may lead into treatment failure. Two different studies in India have contrast in their finding where Potron in 2011 has found that blaKPC-2 encoding plasmid was non typeable while performing PCR based replicon typing [12]. Though later Kumaraswamy in 2011 described that enterobacteriaceae (Klebsiella and E. coli) were harboring some resistance gene within inc $A / C$ type plasmid [13]. In this study it was observed that this centre, $\mathrm{F}$ inc type was more predominant followed by I and $\mathrm{Y}$ inc type. Also, it is evident from current investigation $\mathrm{F}$ inc type plasmids were encoding multiple resistance gene and could propagate within broad host range. Thus, this inc group could possibly responsible for horizontal transmission of multidrug resistance, their maintenance, persistence within hospital isolates under antibiotic stress.

Klebsiella pneumoniae that produced 16S rRNA methylases 113 were reported in 2003 [14]. These enzymes were found to 
Citation: Choudhury NA, Deb S, Maurya AP, Chanda DD, Chakravarty A, et al. (2016) Phenotypic Screening of Aminoglycoside Resistance and their Transferability in Clinical Isolates of Klebsiella pneumoniae from India. J Med Microb Diagn 5: 218. doi:10.4172/2161-0703.1000218

confer extraordinarily high levels of resistance to clinically useful aminoglycosides, such as amikacin, tobramycin, and gentamicin [15].

\section{Conclusion}

Aminoglycosides are used to treat both gram positive and gram negative bacterial infections. This group of antibiotic is considered to be a second line drug which is prescribed in combination with other groups in clinical settings. Carriage of aminoglycoside resistance and their horizontal transferability in hospital setting demands urgent need to devise proper antibiotic policy and to slow down their expansion from hospital to communityenvironment.

\section{Acknowledgement}

The authors would like to acknowledge the help of HOD, Microbiology, Assam University for providing infrastructure. The authors sincerely acknowledge the financial support provided by Maulana Azad National Fellowship to carry out the work.

\section{References}

1. Hirsch EB, Tam VT (2010) Detection and treatment options for Klebsiella pneumoniacarbapenemases (KPCs): an emerging cause of multidrug-resistant infection. review, J Antimicrob Chemother 1119-1125

2. Magnet S, Blanchard JS (2005) Molecular insights into aminoglycoside action and resistance. Chem Rev 105: 477-98.

3. Vakulenko SB, Mobashery S (2003) Versatility of aminoglycosides and prospects for their future. Clin Microbiol Rev 16: 430-450.

4. Thomas RF, Castanheira M, Miller GH, Jones RN, Armstrong ES (2008) Detection of Methyltransferases Conferring High-Level Resistance to Aminoglycosides in Enterobacteriaceae from Europe, North America, and Latin America. Antimicrobial Agents and Chemotherapy 1843-1845.

5. Clinical Laboratory Standard Institute (2011) Performance Standards for
Antimicrobial Susceptibility Testing; Twenty-First Informational Supplement M100-S21 CLSI, Wayne, USA.

6. Li S, Anderson ML, Yang H (2007) Heat shock method, Applied Physics Letter 91, 013902.

7. Gelder LD, Ponciano MJ, Joyce P, Top EM (2007) Stability of a promiscuous plasmid in different hosts: no guarantee for long term relationship. Microbiology 153: $452-463$.

8. Carattoli A, Bertini A, Villa L, Falbo B (2005) Identification of plasmids by PCRbased replicon typing. Journal of Microbiological Methods (63rdedn) 219-228.

9. Angelica R, Helia B, Mariana D, Mella S, Zemelman R, et al. (2003) Prevalence and types of class 1 integrons in aminoglycoside-resistant Enterobacteriaceae from severalChilean hospitals. Journal of Antimicrobial Chemotherapy 51: 317 321.

10. Ma L, Lin CJ, Chen JH, Fung CP, Chang FY, et al. (2009) Widespread Dissemination of Aminoglycoside Resistance Genes armA and rmtB in Klebsiella pneumoniae Isolates in Taiwan Producing CTX-M-Type ExtendedSpectrum Lactamases, antimicrobial agents and chemotherapy 53: 104-111.

11. Gelder LD, Williams JJ, Ponciano JM, Sota M (2008) Adaptive plasmid evolution results in host-range expansion of a broad-host-range plasmid. Genetics 178 : 2179-2190.

12. Potron A, Poirel L, Verdavaine D, Nordman P (2011) Importation of KPC-2producing $E$. coli from India. Journal of antimicrobial chemotherapy.

13. Kumarasamy K, Kalyansundaram A (2012) Emergence of Klebsiella pneumonia isolate co-producing NDM-1 with KPC-2 from India. Journal of chemotherapy 67: $243-244$

14. Galimand M, Courvalin P, Lambert T (2003) Plasmid-mediated high-level resistance to aminoglycosides in Enterobacteriaceae due to 16S rRNA methylation. Antimicrob Agents Chemother 47: 2565-2571.

15. Doi Y, Arakawa Y (2007) 16S Ribosomal RNA Methylation: Emerging resistanceMechanism against Aminoglycosides. Clinical Infectious Diseases 45: 88-94. 